\title{
Implementation issues in RFID-based Anti-counterfeiting for Apparel Supply Chain
}

\author{
Choi S.H. ${ }^{1} \quad$ Cheung H.H. $^{2} \quad$ Yang B. $^{3} \quad$ Yang X.Y. $^{4}$ \\ Department of Industrial and Manufacturing Systems Engineering, \\ The University of Hong Kong, Pokfulam Road, Hong Kong
}

\begin{abstract}
With advancement in Radio Frequency Identification (RFID), RFID-based track-and-trace anti-counterfeiting has attracted considerable research interests. Track-and-trace anticounterfeiting requires a trustworthy electronic pedigree (e-pedigree) to ensure high product visibility. Continuous movements of large volumes of products along the supply chain would generate huge amounts of data, posing great challenges to system development and operation. As such, the front-end RFID data should be well-formatted to facilitate data capturing, filtering, and synchronization, so that the accumulated e-pedigree is complete and trustworthy for subsequent product authentication.

In this paper, we present an innovative track-andtrace anti-counterfeiting system for apparel products, and discuss some key implementation issues, such as e-pedigree formatting, tag data processing, data synchronization, and traceability / visibility control.

An e-pedigree data format for full traceability of apparel is proposed to support products authentication at item-level, products anti-lost at pallet-level, and products status prediction at batchlevel. With this format, an automatic data synchronization mechanism is established to ensure e-pedigree integrity. This mechanism is integrated with a Tag Data Processing and Synchronization (TDPS) algorithm to create unique product identifiers (PID) for tagging individual apparel items and subsequently synchronize the e-pedigree and related data.

Moreover, a parent-and-child containment relationship between items and cartoons is established and stored in the back-end database servers to maintain accuracy and integrity of the $e$ pedigree when mis-reading occur at product receiving at supply chain nodes.

Lastly, to avoid possible leakage / falsification of e-pedigree data, an RBAC-based access control is proposed as an auxiliary module for the anticounterfeiting system.
\end{abstract}

\section{Introduction}

Product counterfeiting is an illicit practice of copying a genuine item to create a fake version for profit. It poses huge threats to manufacturing industries and the global economy. The number of counterfeit products has been skyrocketing in recent years. The International Anti-Counterfeiting Coalition (IACC) [1] estimates that $\$ 600$ billion is lost annually due to counterfeiting. This has called for a reliable anti-counterfeiting technology to safeguard authentic products, to help companies fight illicit competition, and to protect the interest of endconsumers.

Current anti-counterfeit technologies can be broadly categorized as follows: 1) Overt, or visible features; 2) Covert, or hidden markers; 3) Forensic techniques; 4) Serialization / Track-and-Trace systems.

The principles and detailed comparisons of these anti-counterfeiting approaches are elaborated in $[2][3][4][5]$. Among them, the track-and-trace approach is found outstanding in combating counterfeiting. In particular, the track-and-trace approach is distinguished by its ability to protect the whole supply chain against infiltration and abuse, as well as the additional benefits in enhancing the supply chain efficiencies, eliminating theft and fraud, facilitating recall of defective products and remote authentication.

Track-and-trace anti-counterfeiting may be based on barcodes (linear or 2D matrix) or RFID tags for identification of product items.

An RFID tag comprises of an antenna within a microchip, which contains specific item-level product information. The information can be interrogated at a certain distance by RFID readers through electromagnetic waves.

Compared with barcode, RFID tends to be much more advantageous, in that it is capable of automatically identifying / authenticating products with non-line-sight, and that it supports fast and massive reading.

With the increasing popularity of RFID in supply chain management during the past decade, a number of RFID-based traceability systems have been established in [6][7][8][9]. However, these systems tend to target at some specific applications of supply 
chain management, such as inventory control, eliminating wastage, and fine grain product recalls.

The concept of utilizing RFID tags to track and trace products for anti-counterfeiting through the whole supply chain was first proposed in [10] and analyzed in [11][12][13][14]. With the evolvement of RFID technology and maturity of item-level applications, some researchers have proposed detailed system architectures [10][15][16] to combat counterfeiting. However, few have been implemented in practice. Indeed, there still exist a number of practical problems that have yet to be solved, especially the issues of data management.

As RFID-tagged items flow through the supply chain, the track-and-trace anti-counterfeiting system dynamically generates a large amount of data about the product movements [17], which forms the skeleton of the product e-pedigree stored in the backend databases.

Considering the importance of e-pedigree for anticounterfeiting, three key issues, namely Pedigree Data Formatting, Pedigree Data Processing, and Pedigree Transmission Mechanism, have to be addressed accordingly [18].

Pedigree Data Formatting is fundamental for epedigree data collection and management. A more expressive format would definitely better facilitate product ID generation, item tracking, authenticating and monitoring.

Pedigree Data Processing refers to the procedure or mechanism used to update / synchronize epedigree data to keep its integrity. Indeed, the reliability of a track-and-trace anti-counterfeiting system hinges largely on the e-pedigree data synchronization.

Pedigree Transmission Mechanism is to control access to the pedigree data, as required for internal / legal / government audit. In fact, this issue calls for a proper data visibility mechanism to deal with several possible high-level scenarios, such as government inspection and after sale processing. These scenarios involve human interactions with the anticounterfeiting system and thus should be wellcontrolled to avoid data leakage / falsification; otherwise the entire system may be corrupted.

Overall, irrespective of the detailed architecture of a track-and-trace anti-counterfeiting system, the data management issues of e-pedigree, such as data formatting and synchronization, have to be fully addressed.

This paper attempts to address these key implementation issues, using apparel supply chain in practice.

We firstly design an expressive e-pedigree data format for apparel products, and then establish a data processing and synchronization mechanism for maintaining e-pedigree integrity. At last, a rolebased access control method is used to control the data visibility for human (internal employees / government) interaction. This paper is organized as follows:

The paper first presents the system architecture of an innovative RFID-based track-and-trace anticounterfeiting system in section 2. Based on this, the data format for e-pedigree of apparel products is proposed in section 3. An automatic data processing and synchronization mechanism is then elaborated in section 4, while data visibility control is explored in section 5. Lastly, a conclusion is drawn and future work discussed in section 6 .

\section{A proposed RFID-based track-and- trace anti-counterfeiting system}

We proposed in our previous work [15] an RFIDbased track-and-trace anti-counterfeiting system for relatively high-end products. The system architecture is shown in Figure 1.

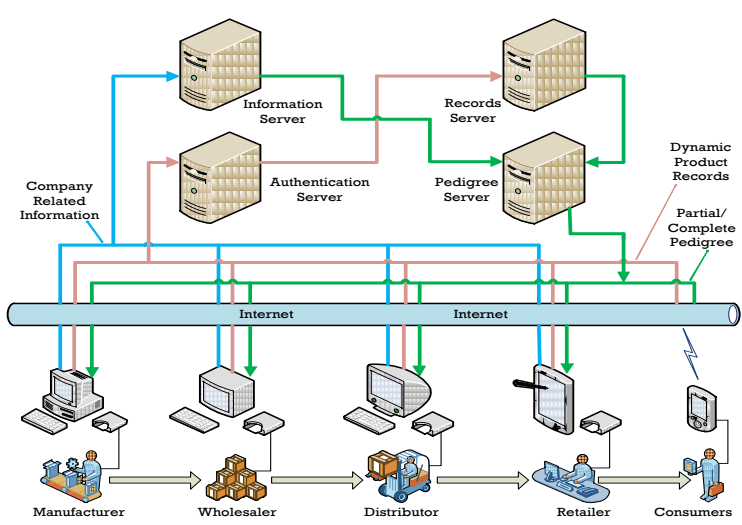

Figure 1. Flow of RFID-based track-and-trace anti-counterfeiting

It mainly consists of two layers, namely a frontend RFID-enabled layer for tag programming and product data acquisition, and a back-end anticounterfeiting layer for processing and synchronization of product e-pedigree and authentication.

The back-end layer consists of a set of computer servers which together enforce track-and-trace anticounterfeiting. The Information Server collects company related information from the supply chain partners through a system registration module. The information is crucial to the product pedigree because it forms the basic geographical picture of the product movement history in supply chain; it also provides the basis for tracing problems when suspected counterfeits appear.

Each product is identified by an embedded RFID tag, which is programmed with a unique product identifier (PID) during production. The PID forms the basis of transaction records, which is sent to the Authentication Server.

The Authentication Server verifies the transaction records and screens out suspicious activities. The screened records are then sent to the Record Server for storage and subsequent follow-up. The supply 
chain partners can verify the partial product pedigree from the point of manufacturing to the previous owners by making requests to the Pedigree Server, which in turn retrieves transaction records from the Records Server as well as company information from the Information Server to generate the required pedigree. They should reject any products with a suspicious partial pedigree.

The Pedigree Server is also responsible for generating complete product pedigrees, through the Internet and the mobile phone network, to endconsumers for verification. When a customer is satisfied with the authenticity of a product and then pay for it, the retailer should generate a sale record, which is subsequently sent to the Authentication Server. Any further transactions of the same product after the sale record are deemed suspicious.

The front-end layer mainly controls RFID devices to read (program) data from (to) the tags attached to product items for processing the data together with the related product information. This layer is particularly crucial to establishing and ensuring accurate e-pedigrees that record all transactions of each of the product items moving along the supply chain, from the manufacturing source to the retail stores.

\section{Format of e-pedigree data for apparel products}

Based on the system architecture of the proposed track-and-trace for anti-counterfeiting described in section 2, we now outline the issue of e-pedigree data formatting and then propose a structure to standardize apparel product e-pedigree information.

In apparel supply chain, each garment item is associated with a Class-1 Generation-2 (C1G2) UHF RFID tag, which stores a PID by combining a programmable 96-bit unique EPC with a 64-bit hardcoded tag unique identifier (UID) during manufacturing, as shown in Figure 2.

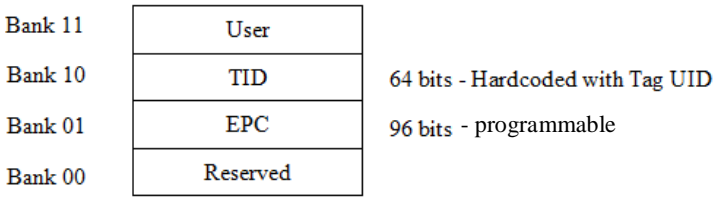

Figure 2a. Memory structure of a C1G2 tag hardcoded with UID in Bank 10

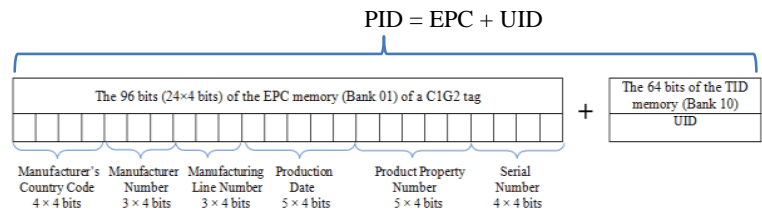

Figure 2b. Customized closed-loop EPC numbering scheme and PID for apparel products
In Figure 2a, the memory of a C1G2 UHF RFID tag can be logically grouped into four memory banks, namely Bank 00 for Reserved memory, Bank 01 for EPC memory, Bank 10 for TID memory which is normally hardcoded with the UID of the tag, and Bank 11 for User memory.

Figure $2 \mathrm{~b}$ shows a customized closed-loop numbering scheme for EPC that uses the 96 bits of the EPC memory in six groups.

The first group has 16 bits for four hexadecimal digits that represent the manufacturer's country code; the second group has 12 bits for three hexadecimal digits to represent the factory code; the third group has 12 bits for three hexadecimal digits to represent the manufacturing / packaging line number; the fourth group has 20 bits for five hexadecimal digits to represent production date; the fifth group has 20 bits for five hexadecimal digits to represent product property; and the final group has 16 bits for four hexadecimal digits to represent a unique serial number for an apparel item produced on a particular line in a particular factory on a particular production date.

As such, the PID formed for an apparel item by combining EPC with UID is unique and informative, as well as resistant to cloning. In particular, it serves as a pointer to the e-pedigree data subsequently accumulated in back-end databases. These data should be standardized, concise and comprehensible such that the accumulated e-pedigree is informative and convincing for product authentication. Otherwise, the whole concept of track-and-trace anticounterfeiting would collapse. Several international workgroups have been developing methodologies of data standardization for applications like food trackand-trace check in EU [19], and Can-trace data standard in Canada [20]. In the following section, we propose a structure to model apparel product epedigree data according to the proposed system architecture.

An appropriate e-pedigree structure that facilitates full traceability of a product requires information of its total lifecycle. This information can be mainly divided into three categories according to the fourelement structure for traceability [18]: (1) static data on logistics units; (2) static data on individual item features (PID); and (3) traceable data on product status changes (e.g. transaction records).

As mentioned in section 2, static data of logistics units includes all the information of the related supply chain companies which lays the foundation of the product e-pedigree. The PID of a product item simply contains the product's feature information, which serves as a pointer to the accumulated epedigree data. Traceable product data consist of item-level release records, transaction records, sale records, pallet-level containment relationship records, and batch-level order information. An 
indicative categorization of e-pedigree data for apparel products is summarized in Table 1.

The proposed e-pedigree data structure is designed to support full product track-and-trace anticounterfeiting for apparel supply chain. It can be rearranged into two parts, namely PID and Traceable Info, as shown in Figure 3. Item PID serves as a pointer that points to historical data (company information, records, containment relationship, etc.) and future state (downstream company information).

\begin{tabular}{|c|c|c|}
\hline $\begin{array}{c}\text { E- } \\
\text { pedigree } \\
\text { Data } \\
\text { Structure }\end{array}$ & Data Field & Description \\
\hline \multirow{5}{*}{$\begin{array}{l}\text { Company } \\
\text { Related } \\
\text { Data }\end{array}$} & $\begin{array}{l}\text { company } \\
\text { name }\end{array}$ & $\begin{array}{l}\text { A logistics unit in apparel supply } \\
\text { chain. }\end{array}$ \\
\hline & $\begin{array}{l}\text { company } \\
\text { type }\end{array}$ & $\begin{array}{l}\text { The type may be manufacturer, } \\
\text { wholesaler, retailer, etc. }\end{array}$ \\
\hline & address & $\begin{array}{l}\text { It is crucial to forming a } \\
\text { geographical map for product } \\
\text { movement in apparel supply } \\
\text { chain. }\end{array}$ \\
\hline & status & $\begin{array}{l}\text { It indicates the validation of the } \\
\text { company. }\end{array}$ \\
\hline & $\begin{array}{l}\text { contact } \\
\text { person }\end{array}$ & For management. \\
\hline \multirow{2}{*}{$\begin{array}{l}\text { Unique } \\
\text { PID } \\
\text { (RFID } \\
\text { tag) } \\
\end{array}$} & TID & $\begin{array}{l}\text { It contains the UID of a tag, which } \\
\text { is factory-locked and difficult to } \\
\text { clone. }\end{array}$ \\
\hline & EPC & $\begin{array}{l}\text { It contains information about } \\
\text { garment colour, size, etc. }\end{array}$ \\
\hline \multirow{6}{*}{$\begin{array}{l}\text { Product } \\
\text { Traceable } \\
\text { Records }\end{array}$} & $\begin{array}{l}\text { release } \\
\text { record }\end{array}$ & $\begin{array}{l}\text { It includes the PID of a product } \\
\text { item, companies involved, time of } \\
\text { record, etc. (for manufacturers) }\end{array}$ \\
\hline & $\begin{array}{l}\text { transaction } \\
\text { record }\end{array}$ & $\begin{array}{l}\text { It includes the PID of a product } \\
\text { item, companies involved, time of } \\
\text { transaction, etc. }\end{array}$ \\
\hline & sale record & $\begin{array}{l}\text { It includes the PID of a product } \\
\text { item, companies involved, sale } \\
\text { time, etc. (for retailers) }\end{array}$ \\
\hline & $\begin{array}{l}\text { containment } \\
\text { relationship } \\
\text { record }\end{array}$ & $\begin{array}{l}\text { It includes relationship between } \\
\text { items and cases, cases and pallets, } \\
\text { etc. }\end{array}$ \\
\hline & order record & $\begin{array}{l}\text { It serves as advanced shipping } \\
\text { notes for tracking of product } \\
\text { status. }\end{array}$ \\
\hline & $\begin{array}{l}\text { mark fake } \\
\text { record }\end{array}$ & $\begin{array}{l}\text { It marks a record in a blacklist of } \\
\text { suspicious items detected by the } \\
\text { system. }\end{array}$ \\
\hline
\end{tabular}

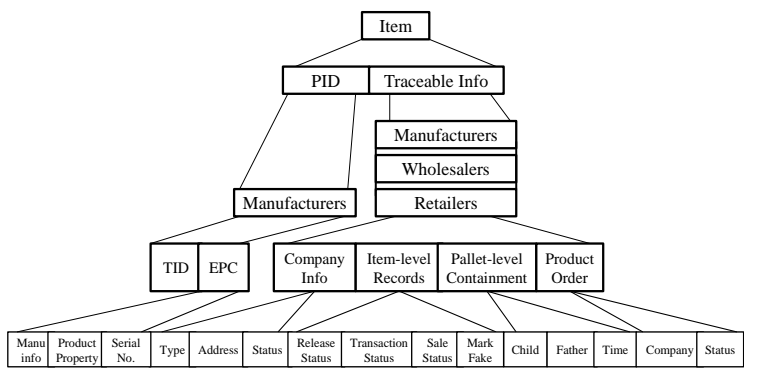

Figure 3. Data structure of apparel product e-pedigree

\section{Mechanism for e-pedigree data processing and synchronization}

As mentioned earlier, the track-and-trace method for anti-counterfeiting hinges on reliable data creation, updating, and synchronization between the front-end supply chain partners and the back-end databases, so that the data on physical movement of all items are recorded in the e-pedigree.

However, without an effective mechanism with proper logics for this purpose, the product e-pedigree can be deemed incomplete and dubious. As a result, fake injection cannot be effectively prevented, and the authenticity of a product item becomes unconvincing even if it has a genuine release record from the factory line.

In our proposed RFID-based track-and-trace anticounterfeiting system, the back-end servers individually or cooperatively process data creation, updating, and synchronization. In general, it can be categorized into three main steps to update and synchronize all the e-pedigree data through Information Server, Authentication Server and Pedigree Server, namely Company Information Collection, Product Release Record Synchronization and Product Transaction Updating. The following sections explain each step in details.

\subsection{Company information collection}

When a supply chain company (a manufacturer, distributor, carrier or retailer) joins in the proposed RFID-aided traceability system, it should first access a registration module which collects the company information, such as company type, address, status and contact person. The company address is essential to form a geographical map for product movement in future. The company status is set as Pending by default; only after the System Holder (usually a Corporation Head or an entrusted third-part entity) has duly checked the validation of the registered company would the status change to Active, which indicates successful company registration.

After this, the registered company is assigned a unique ID, with which its contact person is able to add peers and access the system to process front-end tagged apparel products, which goes to the second step. Company Registration is mainly processed by Information Server in the Figure 4a through the interface in Figure 4b.

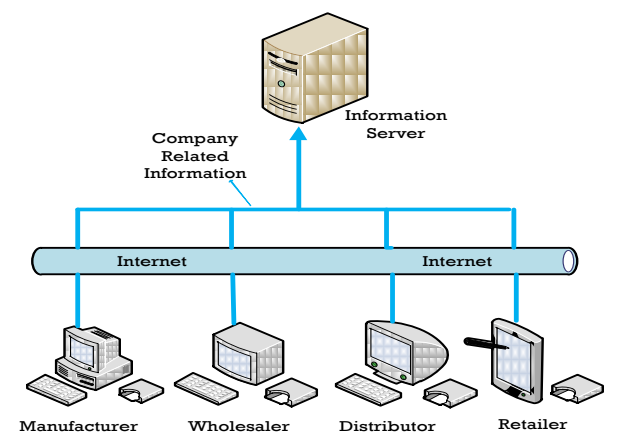

Figure 4a. Flow of company information collection 


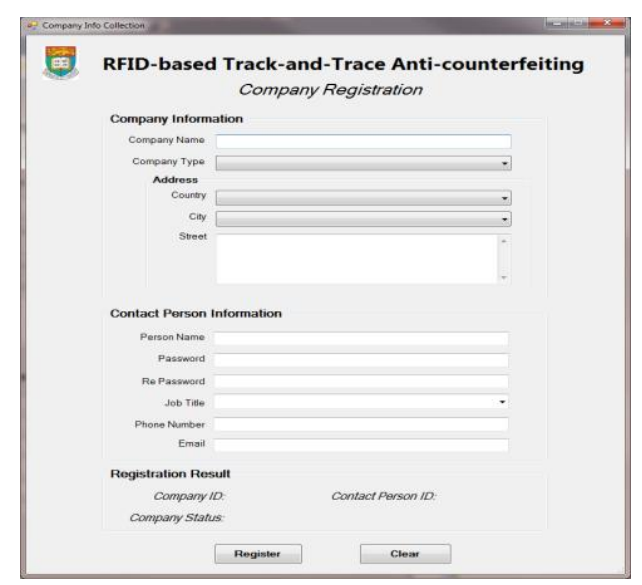

Figure $4 \mathrm{~b}$. Interface for company information collection

\subsection{Product release record synchronization}

The second step of data processing and synchronization is designed for manufacturers to create a PID of a tagged item and synchronize product release information during production. Before production starts at a front-end manufacturing line, an Active manufacturer is firstly required to access the system to obtain the right to update item Release Records.

When a product is completed (including real-time RFID tag programming unique 96-bit EPC into Bank 01), a Release Record is composed and then updated to the Authentication Server for storage. The status of that product is then set as Released. This forms an initial e-pedigree of that item and nobody has the right to change or delete it. Tagged items are packaged and distributed based on order requirements from downstream supply chain nodes. The related order information and containment relationship information is similarly updated to the anti-counterfeiting system.

To establish a trustworthy e-pedigree, the PID for each product item must be accurately created and programmed to the tag during production, and then synchronized through the network to be stored in the Authentication Server to facilitate subsequent logistics operations and anti-counterfeiting at downstream nodes of the supply chain.

If the process of data creation and synchronization for the initial e-pedigree information at a manufacturing line is not well designed and controlled, two issues may likely emerge: 1) A genuine product has been produced from the manufacturing lines, but there are no corresponding PID / e-pedigree data in back-end servers; and 2) There are e-pedigree data (redundant data), but no corresponding item movement through the supply chain.

Apparently, these two issues would likely corrupt the track-and-trace system because some genuine products may be treated as illicit items while redundant e-pedigree data may be maliciously used by counterfeiters.

Specifically, the process of creating the PID of a tagged product item and hence an initial e-pedigree at a manufacturing / packaging line involves realtime execution of tag programming, UID reading, locking, verification, and data encryption and synchronization to the back-end databases.

As discussed in our previous work [21], it is not an easy task to program all required information accurately into a tag and then lock the Bank 01 and update the tag data to the back-end databases in real time, particularly when the product items are being transferred at a relative high speed to avoid affecting the production rate.

The speed at which a tag is moving when it is being programmed and locked is called the "tag moving speed". Two common programming errors, namely partial tag programming and duplication error, are often encountered [21]. Such errors would affect trustworthiness of a product e-pedigree and integrity of the supply chain.

To alleviate the problems related to tag data processing and synchronization, a Tag Data Processing and Synchronization (TDPS) algorithm for integration with an RFID-based apparel packaging line as in Figure 5, is proposed for controlling RFID readers to program a unique 96-bit EPC to Bank 01 of a specific tag, lock the Bank 01, verify the result of tag programming and locking, and then encode the data to create the production record and initialize the e-pedigree.

The proposed TDPS algorithm mainly consists of five steps, including: 1) Tag EPC Programming, 2) EPC Verification and UID Reading, 3) Tag Locking, 4) Locking Verification, and 5) Storing PID and Creating Initial E-pedigree, as shown in Figure 6.

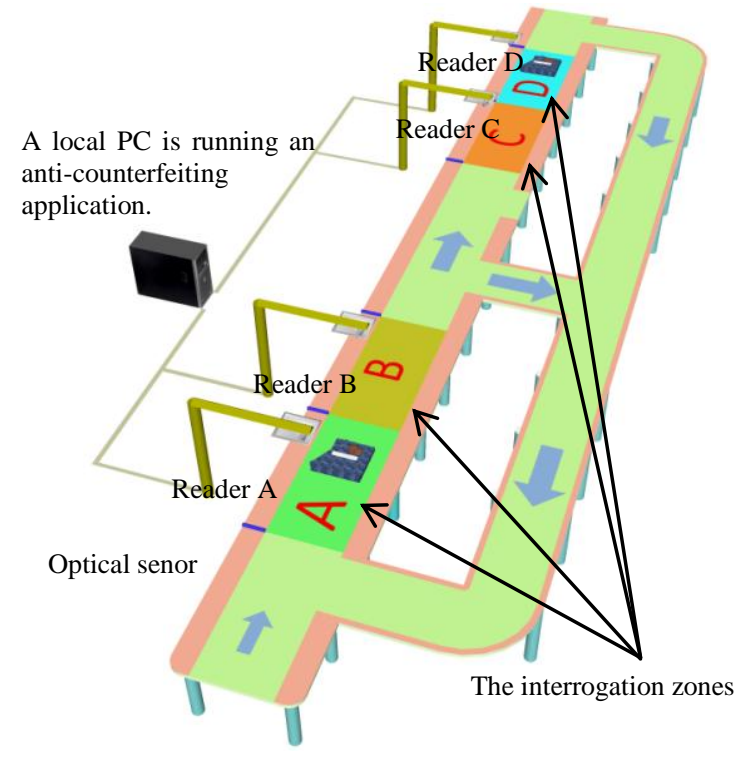

Figure 5. The TDPS algorithm integrated with an RFIDbased apparel packaging line 
Figure 5 shows an RFID-based apparel packaging line mainly contains RFID readers, optical sensors, and an anti-counterfeiting application running on a local PC which controls readers to process tag EPC programming, reading, locking, and verification, respectively when an apparel item enters into various interrogation zones.

As discussed in our previous work [21], an RFID reader communicates randomly with all tags in range when tagged product items continuously pass one by one through an interrogation zone of the reader during mass production of the product items. It would not be practicable to selectively write a specific EPC number to a particular tag, especially when the product items are moving a high speed and being close to one another.

To overcome this challenge, a filtering strategy was implemented to prevent repeated tag programming and the details were presented in [21]. For simplification, the proposed RFID-based apparel packaging line is configured to line up packaged apparel items one by one without stacking. As such, a target tag can be singulated in the interrogation zone to facilitate correct tag programming and locking by controlling output RF power from the reader and adjusting the separate distance between tagged apparel items without compromising the production rate.

The following section elaborates the step of the TDPS algorithm for establishing a trustworthy epedigree at the RFID-based apparel packaging line.

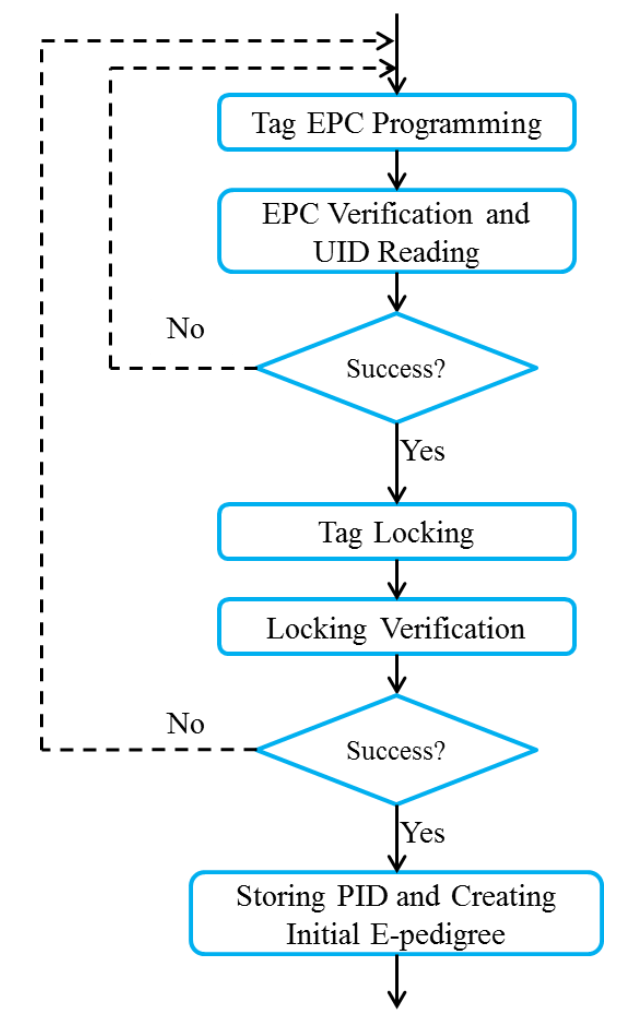

Figure 6. TDPS algorithm in a manufacturing / packaging line
Step 1: The Tag EPC Programming step controls a reader to write a unique 96-bit EPC to Bank 01 of a specific tag when a product item is detected entering into the interrogation zone. In this step, the critical issue is to generate the unique EPC for each product and write it accurately to the tag. A local anticounterfeiting application firstly composes the Manufacturer's Country Code, Manufacturer's Number, and Manufacturing Line Number. These data are generated when the manufacturer logs in the system in a certain manufacturing line. Secondly, the Product Property Number is generated based on the product information that could be accurately pretyped into the system by the operator for each batch of production. At last, the Production Date may be taken from the system clock, while the Serial Number is composed in real-time to form the unique EPC. After successful tag EPC programming of a product item, the Serial Number is automatically increased by one for the next item. In practice, however, tag EPC programming may fail due to various reasons, particularly insufficient RF power to perform write operation communication between the reader and tag caused by the fast tag moving speed and environmental disturbances. Hence, it is imperative to validate whether or not the 96-bit EPC has been successfully written to the tag.

Step 2: After finishing tag EPC programming of a product item, the EPC in the tag memory Bank 01 and the UID in Bank 10 of the tag are read to form the PID. Indeed, before locking Bank 01 and storing the PID to the Authentication Server, it is necessary to verify successful writing of EPC into Bank 01. A method for verification of EPC programming is to read the 96-bit EPC of the tag and then compare it with the target EPC generated in step 1. Tag EPC programming is considered successful only if the EPC read from the tag match exactly with the target EPC. However, this method is not reliable, because reading 96-bit EPC is time-consuming, particularly when the tag is moving together with a product item moving at a relatively high speed. According to our previous work [21], it is often found that a portion of data cannot be written fully to the last several 16-bit word memory of Bank 01 of a tag being processed in a production line because of insufficient RF power caused by fast tag moving speed and environmental disturbances. For example, the 16-bit memory from $70 \mathrm{~h} 7 \mathrm{Fh}$ has higher chances of failed writing than the 16-bit memory from 20h 2Fh of Bank 01, as shown in Figure 7. This is because a Write command writes one word (i.e. 16 bits) at a time and the writing operation starts at address from $20 \mathrm{~h} 2 \mathrm{Fh}$ first according to GEN2 standards [6]. This means there is not enough time for the reader to write data into the remaining memory of Bank 01 when the tag is moving fast. 


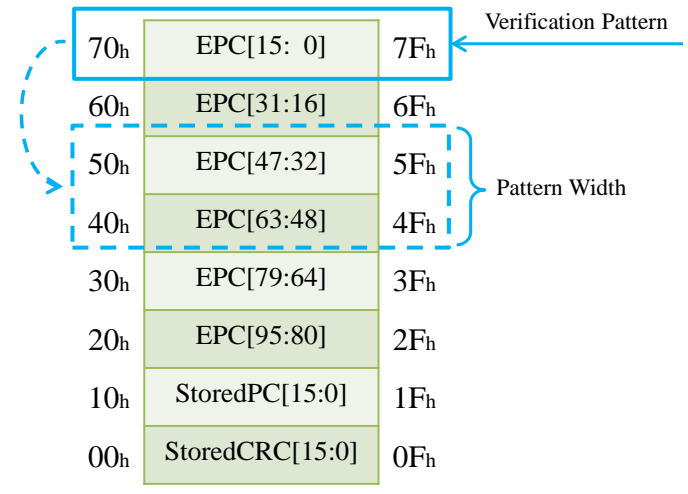

Figure 7. Verification Pattern on Bank 01

Based on this phenomenon, we propose a verification pattern to verify the EPC writing result by partial reading of the EPC bits from the end addresses of Bank 01 for comparison with the corresponding bits of the target EPC. Tag EPC programming is considered successful if all these bits match. This verification pattern can greatly shorten the reading time so critical to the whole tag processing cycle when the tag is moving at a high speed. For example, a practicable verification approach is to read only the last 16 bits from Bank 01 , and then verify them with the corresponding 16 bits of the target EPC. However, it should be pointed out that the verification accuracy may drop when fewer bits are read for comparison, despite saving of reading time. It is therefore necessary to balance the time consumption and the verification accuracy to enhance the efficiency of the TPDS algorithm. Figure 7 shows the verification pattern on Bank 01 . The width and the starting position of the pattern may be adjusted from the last 16-bit memory to the first 16-bit EPC memory. With different widths and starting positions, the pattern should have different time consumption and verification accuracy. Based on this mechanism, the optimal verification pattern could be determined based on the design requirements of the RFID-based packaging lines for various kinds of products. After finishing this step, the item would be transferred forward for further processing if it has been successfully programmed; otherwise it would be cycled back for reprogramming, as shown in Figure 6.

Step 3: After finishing EPC verification, each tag needs to be locked; otherwise, the genuine tag information could be easily and maliciously changed when the product items move in the supply chain. GEN2 tags have four types of locking status, namely 1) Unlocked (None), 2) Perma-unlocked (can never be locked), 3) Locked, and 4) Perma-locked (can never be unlocked). Locked tags cannot be changed without presenting a valid 32-bit Access Password, while Perma-locked tags cannot be changed permanently. Considering possible recycling of RFID tags in future, Locked type is adopted in the
TPDS algorithm. For simplification, a constant 32bit Access Password is assigned to each batch of products and the Password is encrypted and then stored in the Authentication Server; the Password is firstly programmed to Bank 00 of tags for this batch of products. Before programming EPC in the manufacturing line, Bank 00 then locked to prevent hackers from reading the Access Password. However, similar to EPC programming, tag locking may also fail due to the fast tag moving speed and environmental disturbances. Tag Locking Verification is therefore required to confirm the locking status.

Step 4: Theoretically, a GEN2 tag which is successfully locked should backscatter a reply within $20 \mathrm{~ms}$ after receiving the Lock command from a reader. If the reader does not receive a reply within $20 \mathrm{~ms}$, the Lock is treated as a failure. However, a fast moving tag may leave the reading range before completing the wireless communication with the reader. Besides, if the reader does not receive the reply from the tag, it cannot declare Lock failure the dynamic environment and fast moving speed may destroy the communication between the tag and the reader. To enhance the robustness of Locking Verification, a method for verification of locking is proposed to continuously write the previously generated unique EPC to the tag again. If one of the Write commands is successful, then the tag is definitely not locked; only that all Write commands fail indicates the tag is well locked. In fact, this Locking Verification method seems to emulate the possible tag falsification attacks. After verification, the item would be transferred forward for further processing if its tag has been successfully locked, while the product which is not locked may be moved back for Tag Programming again. At the meantime, the EPC and UID combined as a PID will be stored to the Authentication Server and an initial e-pedigree is created and recorded at the final step.

Step 5: After successfully finishing EPC Programming, EPC Verification and UID Reading, Tag Locking, and Locking Verification, the EPC and the UID are combined to form the PID of a tagged product item. The PID is recorded accordingly in the release record to initialize the product e-pedigree, which is synchronized to the Authentication Server. The detail of this release record has been elaborated in section 3 and can be found in Table 1 . The product status is marked as "Released" in this first e-pedigree record, to which no further modification is allowed. However, during the process of data synchronization, two issues may arise. First, broken network connection or network congestion may prevent epedigree updating. Second, malicious network analyzers or packet sniffers may capture the genuine product information if the data are not encrypted. A possible approach to alleviating these problems is to apply asymmetric cryptography to encrypt the e- 
pedigree and to temporarily store it in cache. Figure 8 shows the detail mechanism of Release Record updating. The Release Record is firstly encrypted using a public key before being directly sent to the Authentication Server. If the internet connection is broken or blocked, the corresponding records will then be temporarily stored in a local cache. Once the connection recovers, the caching records would be automatically updated again. After receiving the encrypted release records, the Authentication Server would use the corresponding private key to decrypt and authenticate the release records before storing them in the database.

The proposed TPDS algorithm can solve a number of problems, including partial EPC writing, duplicate EPC programming, un-locking, failure of e-pedigree updating due to broken network connection or network congestion, which in practice are commonplace during the processes of tag programming, locking, and data synchronization.

More importantly, it can be adapted to suit various design requirements and configurations of RFID-based packaging lines to achieve a good balance between tag moving speed and tag programming and locking rate, essential for generating PIDs of individual product items and establishment of a trustworthy e-pedigree for supply chain management.

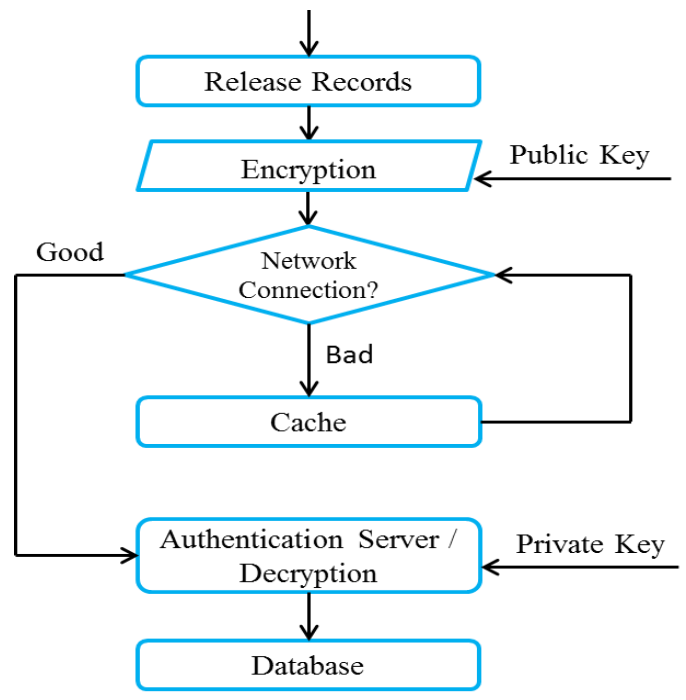

Figure 8. Release Record Updating Mechanism

\subsection{Synchronization of product transaction records}

After production in the factory, product items are transported to downstream supply chain nodes, such as wholesalers, distributors, and retailers. These partners mainly shoulder the responsibility to synchronize product transaction information, as in the third step.
When products are delivered to a warehouse center of a logistics company, the operator is firstly required to identify all the incoming garments utilizing proper RFID equipment (RFID-aided gate door, tunnel, handheld readers, etc.) to authenticate the products accordingly. The detailed authentication mechanism will be elaborated in section 4.4.

Corresponding new Transaction Records would be composed and updated for successfully verified products, while any suspicious items would be screened out and marked as Fake.

Product Containment Relationship may be changed and the items are repackaged for further distribution, according to the specific need of product orders from downstream supply chain nodes.

In retail shops, the cashier generates the final Sale Record when an item is sold by marking the record as Sold once transaction confirmed, after which no further modification for the Sale Record is allowed. Product Transaction Updating is processed by the Authentication Server and the Pedigree Server cooperatively.

However, during synchronization of product transaction data, there is a critical issue. When a batch of items is received by a supply chain partner (wholesaler, distributor or retailer) in a warehouse, RFID miss-reading and error-reading usually happen. This issue affects the completeness and accuracy of a product e-pedigree.

For example, a batch of 500 products is delivered to a wholesaler's warehouse, but only 490 items are checked and synchronized by RFID devices, while the remaining 10 items are missing. The e-pedigree data of these 10 missing items are not updated, and they would arouse suspicion in any further transactions because of their incomplete e-pedigree. This would definitely affect the benefits of supply chain partners and the integrity of the track-and-trace anti-counterfeiting system.

A method to deal with the above issue is to take advantage of the product containment relationship information. From the packaging line in a factory, products are all tagged at item-level, carton-level, and pallet-level. After being packaged, each item PID is associated with its carton's ID, and each carton with its pallet's ID. During the data synchronization in each supply chain node, this containment relationship information is also updated.

When products arrive at the warehouse center for checking, some of the items may not be identified due to miss-reading and error-reading. The precreated product containment relationship information stored in the back-end database can help find out all the items which are missed by the RFID reader.

For example, wholesaler $\mathrm{W}$ intends to deliver 50 items to retailer $\mathrm{R}$ and those 50 products are in the same carton, as shown in Figure 9. Once that carton of products are sent out from wholesaler W, a parentand-child containment relationship is then 
established between a specific carton ID and the PIDs of all 50 items. This relationship information is synchronized to the back-end database. When that carton arrives at retailer $\mathrm{R}$, a list of detected tags can be acquired using RFID readers and a list of expected tags can be obtained based on the containment relationship. A list of non-found tags could then be presented by the local anticounterfeiting system. It should be noted that the non-found products may be stolen by someone during transportation, or may be indeed miss-reading (still existing in the carton). In this case, further operations such as opening the carton or using handheld readers to check again may be needed.

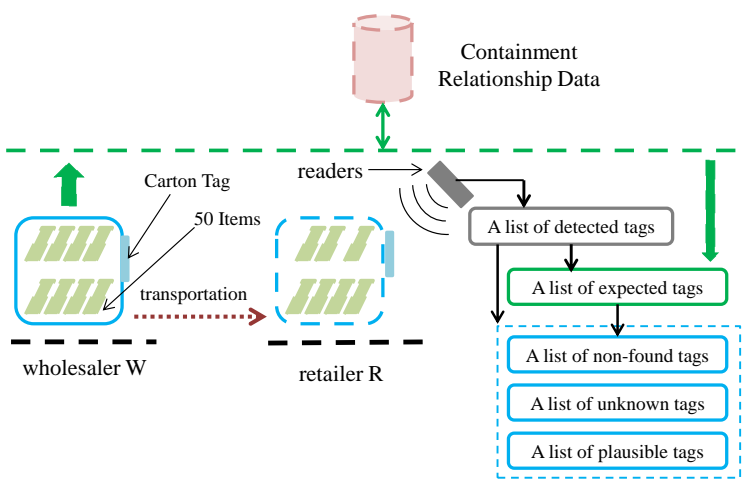

Figure 9. Using containment relationship for processing of miss-reading

The parent-and-child containment relationship information facilitates the process of batch product receiving. If there are either miss-reading or errorreading, the local anti-counterfeiting system will give alerts to operators for further checking. As a result, the accuracy and completeness of a product epedigree can be maintained during the data synchronization in warehouse in the supply chain.

\subsection{Product authentication mechanism}

As mentioned in the previous section, the automatic authentication mechanism executes the core concept of traceability in anti-counterfeiting. Figure 10 shows the detailed logics and procedures. Authentication of a product item consists of four steps.

The first three steps are aimed at checking whether the item has been ever Released by the manufacturer, or whether it has been marked as Fake, or whether it has been Sold before. The last step is to form the partial or whole geographical picture based on the item e-pedigree.

If the result of any step is suspicious or implausible, the product item would be deemed illicit and screened out when it passes the Authentication Zone. Both authentication request and transaction records synchronization are carried through SOAP requests.
Based on the accumulated e-pedigree data, there may be four possible Authentication Results, namely, AR1 represents the status of the upstream company is Active; the item has Release record, plausible transaction records, and the e-pedigree is complete; AR2 means the status of the upstream company is Active; the item has Release record but the e-pedigree is implausible / incomplete; AR3 represents the status of the upstream company is Active; the item does not have Release record; AR4 means the status of the upstream company is not Active, no matter what type of e-pedigree data the item has.

Only cartons containing all product items with AR1 would be accepted and transferred to a warehouse, and the e-pedigree data of those items would then be updated accordingly. Other cartons containing any suspicious, fake, or missing items (even if only one item) would then be transferred to a buffer zone for further processing. The ways of handling these problematic/suspicious products are based on business contracts between the two involved supply chain nodes.

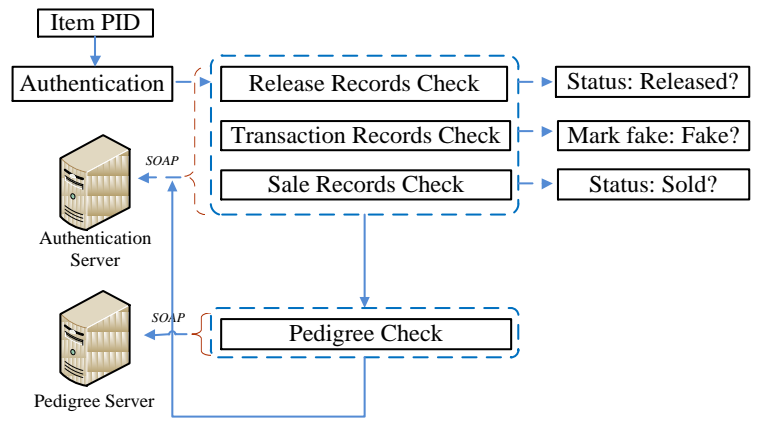

Figure 10. Automatic authentication mechanism

Overall, this synchronization mechanism and the authentication steps are all conducted by the system automatically without any human intervention, thus guaranteeing the credibility of the e-pedigree data and the reliability of the whole anti-counterfeiting system.

\section{RBAC-based data visibility control mechanism}

The synchronized and accumulated product epedigree data in the back-end databases may have to be checked or inspected (from time to time) by internal supply chain practitioners or government auditors for the purpose of after-sale service or legal audit.

In these scenarios, human interactions with the anti-counterfeiting system become inevitable, and such interactions should be well-controlled to avoid possible data leakage / falsification or corrupting the entire system. Hence, a proper data visibility 
mechanism is needed to serve as an auxiliary module for the anti-counterfeiting system. In this section, we introduce a Role-based Access Control (RBAC) method and tailor it for our system.

As a quick review, RBAC was formalized in 1992 by David and Kuhn in [22]. Now the family of RBAC is commonly referred to as the RBAC96 model [23].

The basic concept of RBAC can be described in Figure 11. Permissions including a variety of data operations (like adding, removing, and viewing) are assigned to roles based on specific policy rules rather than directly to individual users; users are assigned to roles rather than directly to permissions. This level of indirection facilitates user-permission management to facilitate system access control.

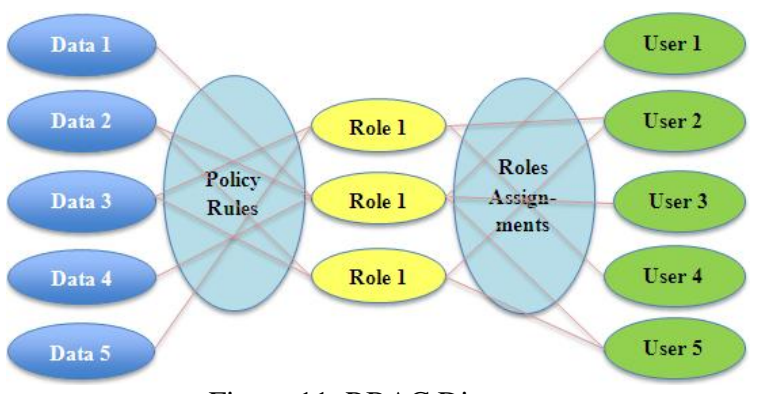

Figure 11. RBAC Diagram

To adopt RBAC for control of human interactions with the anti-counterfeiting system, different roles have to be defined. Each role is assigned a scope of data access / operation tasks. When supply chain employees, government auditors or licit third party outsiders attempt to interact with the back-end databases, those users are dynamically assigned one or more roles. The roles or privileges would be revoked immediately afterwards.

Besides, all of these human interactions would be automatically logged in an individual table by the system. It should be noted that the total number of system roles and the scope of each role are defined based on the practical needs with reference to the apparel supply chain rules and the related business laws.

Overall, by limiting the privilege of accessing back-end databases, this RBAC-based access control module helps safeguard the security of back-end epedigree data, thus preventing possible data leakage / falsification.

\section{Conclusion and future work}

Based on an innovative RFID-based track-andtrace anti-counterfeiting system architecture, we developed a comprehensive data structure for modeling apparel product e-pedigree, together with a mechanism for data creation, updating and synchronization to guarantee the integrity and reliability of product e-pedigree data.

One significant benefit of this data model is the enhancement of track-and-trace capabilities of product movement. By tracking information like item-level transaction records, pallet-level containment relationship, and batch-level order information, the system is able to verify individual items, detect missing/injected objects and predict products' future status at any location of the apparel supply chain.

Based on the e-pedigree data structure, an automatic data processing and synchronization mechanism has been proposed to maintain the product e-pedigree along the whole supply chain. The TDPS algorithm, which is integrated with the mechanism, has been proposed to generate the initial product e-pedigree record when tagged items are processed in a manufacturing / packaging line.

Product containment relationship has been used to deal with the issue of miss-reading, thus facilitating the process of product receiving and authentication in batch-level.

At last, we adopted RBAC mechanism for visibility control of e-pedigree data to prevent possible data falsification and data leakage.

Overall, this paper has addressed some major implementation issues in RFID-based track-and-trace anti-counterfeiting for apparel supply chain.

For future work, the proposed e-pedigree data model and the data synchronization / visibility mechanism would be implemented and evaluated in practical deployment settings.

\section{References}

[1] Counterfeiting Coalition (IACC) http://www.iacc.org/ (13 Novenber 2013) .

[2] Report of World Health Organization (WHO): Anticounterfeit Technologies for the Protection of Medicines,

http://www.who.int/impact/events/IMPACTACTechnologiesv3LIS.pdf (13 Novenber 2013).

[3] Li. L. (2013) 'Technology Designed to Combat Fakes in The Global Supply Chain', Business Horizons, 56(2), 2013, pp.167-177.

[4] Lehtonen, M. (2009) 'Description of The Status Quo of Existing Technical Countermeasures: their benefits and shortcomings', Version 1.01.

[5] Bansal, D., Malla, S., Gudala, K. and Tiwari, P. (2012) 'Anti-counterfeit Technologies: A Pharmaceutical Industry Perspective', Scientia Pharmaceutica, 81(1), pp.1-13.

[6] EPCglobal Networks, http://www.gs1.org/epcglobal. (13 Novenber 2013).

[7] Cheung, A., Kailing, K., Schonauer, S. and Theseos. (2007) 'Query Engine for Traceability across Sovereign, Distributed RFID Databases', in Proceedings of the 23rd International Conference on Data Engineering, Istanbul, Turkey.

[8] DIALOG: Distributed Information Architectures for collaborative logistics, http://dialog.hut.fi/. (13 Novenber 2013). 
[9] Främling, K. and Nyman, J. (2009) 'From tracking with RFID to intelligent products', in Proceedings of 14th IEEE International Conference on Emerging Technologies and Factory Automation, Palma de Mallorca, Spain.

[10] Koh, R., Schuster, E.W., Chackrabarti, I. and Bellman, A. (2003) 'White Paper: Securing the Pharmaceutical Supply Chain', Auto-ID Labs, Massachusetts Institute of Technology.

[11] Staake, T., Thiesse, F. and Fleisch, E. (2005) 'Extending the EPC Network-The Potential of RFID in Anti-counterfeiting', ACM Symposium on Applied Computing, pp.1607-1612.

[12] Staake, T., Michahelles, F., Fleisch, E., Williams, J.R., Min, H., Cole, P.H., Lee, S.G., McFarlane, D. and Murai, J. (2008) 'Anti-counterfeiting and Supply Chain Security, Networked RFID Systems and Lightweight Cryptography', pp.33-43.

[13] Kim, J. and Kim, H. (2005) 'Anti-counterfeiting Solution Employing Mobile RFID Environment', World Academy of Science, Engineering and Technology.

[14] Lehtonen, M., Staake, T. and Michahelles, F. (2008) 'From Identification to Authentication-A Review of RFID Product Authentication Techniques', Networked RFID Systems and Lightweight Cryptography, pp.169-187.

[15] Choi, S.H. and Poon, C.H. (2008) 'An RFID-based anti-counterfeiting system', International Journal of Computer Science, 35(1), pp.1-12.

[16] BRIDGE (2009) http://www.bridgeproject.eu/index.php/mainpage/en/ (13 Novenber 2013).

[17] Jansen-Vullers, M.H., van Dorp, C.A. and Beulens, A.J.M (2003) 'Managing traceability information in manufacturer', International Journal of Information Management, 23(5), pp. 395-413.

[18] Cole, P.H. and Ranasinghe, D.C. (2008) Networked RFID Systems and Lightweight Cryptography: Raising Barriers to Product Counterfeiting, Springer, Berlin.

[19] Smart Food and Agribusiness: http://www.smartagrifood.eu/ (16 January 2014).

[20] Can-Trace Development for traceability standards for food products, http://www.can-trace.org/. (13 Novenber 2013).

[21] Cheung, H.H. and Choi, S.H. (2011) 'Implementation issues in RFID-based anti-counterfeiting systems', Computers in Industry, 62(7), pp.708-718.

[22] Ferraiolo, D.F. and Kuhn, D.R. (1992) 'Role-Based Access Controls', 15th National Computer Security Conference, pp554-563.

[23] Sandhu, R.S., Coyne, E.J., Feinstein, H.L. and Youman, C.E. (1996) 'Role-Based Access Control Models', IEEE Computer, 29(2), pp38-47. 